\title{
Application of the 10-moment model to MEMS flows
}

\author{
Yoshifumi Suzuki* and Bram van Leer ${ }^{\dagger}$ \\ Department of Aerospace Engineering \\ University of Michigan, Ann Arbor, MI 48109-2140, USA
}

\begin{abstract}
In this paper we use the 10-moment description of fluid flow to compute internal nozzle flow and external flow around a micro-airfoil in the continuum-transition regime. The numerical method used is Hancock's scheme, a second-order non-oscillatory Godunov-type scheme; the numerical flux function incorporated is of the Harten-Lax-van Leer (HLL) type and due to Linde (HLLL). The numerical solutions are validated by a comparison to results obtained with Navier-Stokes code, a hybrid DSMC/Navier-Stokes method and an experiment. The 10-moment solution is closed to the experimental results and obtained more efficiently than by the particle method, with considerable speed-up still expected from the use of Discontinuous Galerkin (DG) methods.
\end{abstract}

\section{Nomenclature}

$\mathbf{A}_{\mathrm{n}} \quad$ coefficient matrix of primitive equations

c random-velocity vector

$\mathbf{F}^{10}$ flux vector of 10-moment equations

$\mathbf{F}_{\mathrm{n}} \quad$ flux vector outward normal to the cell face

$\mathcal{G}$ Gaussian distribution function

$H(x)$ Heaviside step function

Kn Knudsen Number

$l \quad$ mean free path

M transformation matrix

$M_{U} \quad$ upstream Mach number

MW molecular weight

$n$ number density

n outward unit vector normal to the cell face

$p \quad$ hydrostatic pressure

$\mathbf{P} \quad$ symmetric positive definite matrix in HLLL Riemann solver

$P_{i j} \quad$ generalized stress tensor

$\mathbf{r}_{i} \quad$ centroid of cell $i$

$r_{t} \quad$ throat half-width

$R \quad$ specific gas constant

Re Reynolds number

$s_{i j} \quad$ area of cell face shared by cells $i$ and $j$

$S$ entropy function

$\mathbf{S}^{10}$ source-term vector of 10 -moment equations

$\boldsymbol{u}$ mean-velocity vector

$\mathbf{U}^{10}$ conserved-variables vector of 10-moment equations

$\boldsymbol{v} \quad$ velocity vector

$V \quad$ middle wave speed in HLLL Riemann solver

$\mathcal{V}$ control volume

W vector of symmetrizing variables in HLLL Riemann solver

${ }^{*}$ Graduate Student Research Assistant, Student Member AIAA

$\dagger$ Professor, Fellow AIAA 
$\mathbf{W}^{10}$ primitive-variables vector of 10 -moment equations

$\tilde{\mathbf{W}}$ reconstructed primitive-variables vector

Symbols

$\alpha \quad$ relative strength of middle wave

$\gamma \quad$ ratio of specific heat

$\boldsymbol{\Theta}$ generalized temperature tensor

$\lambda \quad$ wave speed

$\mu \quad$ viscosity

$\rho$ density

$\sigma \quad$ accommodation coefficient

$\tau$ relaxation time

$\phi_{i} \quad$ gradient limiter

$\phi_{i} \quad$ column vector of $\boldsymbol{\Phi}$

$\boldsymbol{\Phi}$ inverse of $\boldsymbol{\Theta}$

$\Delta t \quad$ time step

\section{Introduction}

$\mathrm{C}^{\text {LOW }}$ in or around micro-electro-mechanical systems (MEMS) typically is in the so-called transition regime $\Gamma$ between continuum and free-molecular flow, with Knudsen numbers $(\mathrm{Kn})$ in the range $0.1 \leq \mathrm{Kn} \leq 10$. In this regime the Navier-Stokes equations, even if allowing for slip at a solid boundary, do not describe the flow with sufficient accuracy. Table 1 summarizes the properties of the simplest models available for a reliable description in different ranges of Knudsen numbers. ${ }^{1}$

Table 1. Simplest mathematical model needed in different flow regimes. The full Boltzmann equation (including collisions) is the most complete model and valid in all regimes.

\begin{tabular}{cll}
\hline Knudsen number & \multicolumn{1}{c}{ Assumption } & \multicolumn{1}{c}{ Mathematical model } \\
\hline $\mathrm{Kn} \rightarrow 0$ & continuum (no molecular diffusion) & Euler equations \\
$\mathrm{Kn} \leq 10^{-3}$ & continuum (with molecular diffusion) & Navier-Stokes equations (no-slip B.C.) \\
$10^{-3} \leq \mathrm{Kn} \leq 10^{-1}$ & continuum-transition & Navier-Stokes equations (1st-order slip B.C.) \\
& & Burnett equations (1st-order slip B.C.) \\
$10^{-1} \leq \mathrm{Kn} \leq 10$ & transition & Moment equations \\
& & Burnett equations (2nd-order slip B.C.) \\
$\mathrm{Kn} \gg 10$ & free-molecular flow & Collisionless Boltzmann equation \\
\hline
\end{tabular}

One may always use a method suitable for a higher Kn range, but this comes at a computational penalty. In particular, the Direct-Simulation Monte-Carlo (DSMC) method, a particle-based method, is required for the highest Knudsen numbers, but in the transition regime it has competition from extended-hydrodynamics methods based either on higher-order PDE's or on large sets of first-order moment equations. The DSMC method, stochastic in nature, gives statistical scatter in the produced solutions, and requires a cell size of the order of the molecular mean free path; the PDE-based methods do not. The multi-moment approach has the additional advantage that the equations only include first derivatives, allowing discretization on the narrowest stencils.

In order to avoid using DSMC where it is not required, Sun and Boyd ${ }^{2,3}$ computed the flow over a micro-airfoil using the particle method only in the vicinity of the airfoil, and a Navier-Stokes code farther away. This calls for blending of the two methods in a buffer zone. The present work attempts to solve the same class of flow problems using a multi-moment model everywhere. 


\section{10-moment model}

The description chosen is the 10-moment model, which is the best known and most studied among models that use multiple moments of the Boltzmann equation. This model is based on a Gaussian velocity distribution (Gaussian closure). ${ }^{4}$ The general form of the Gaussian velocity distribution $\mathcal{G}$ is as follows.

$$
\mathcal{G}(\boldsymbol{x}, \boldsymbol{v}, t)=\frac{n(\boldsymbol{x}, t)}{(2 \pi)^{3 / 2}(\operatorname{det} \boldsymbol{\Theta})^{1 / 2}} \exp \left(-\frac{1}{2} \Theta_{i j}^{-1} c_{i} c_{j}\right),
$$

where

$$
\Theta_{i j}=\frac{P_{i j}}{\rho}, \quad i, j \in\{1,2,3\},
$$

$n(\boldsymbol{x}, t)$ is the number density, $\boldsymbol{c}(\boldsymbol{x}, t)$ the random velocity, and $P_{i j}$ the generalized stress tensor. The model is equivalent to the Navier-Stokes equations without heat conduction; this is sufficiently accurate for the flow problem studied, which has an almost isothermal solution.

The 10-moment model is derived as follows. Assume the velocity distribution function used with the Boltzmann equation is Gaussian, $\mathcal{G}$, and integrate over all particle velocities. The Gaussian velocity distribution has the mathematical property that third-order velocity moments are zero (leading to zero heat flux), which leads to closure of the set of moment equations. Using the BGK approximation for the collision operator and expressing the equations in vector form in a 3-D Cartesian coordinate system, the 10-moment transport equations assume the form

$$
\frac{\partial \mathbf{U}^{10}}{\partial t}+\frac{\partial \mathbf{F}^{10}}{\partial x}+\frac{\partial \mathbf{G}^{10}}{\partial y}+\frac{\partial \mathbf{H}^{10}}{\partial z}=\mathbf{S}^{10}
$$

where $\mathbf{U}^{10}$ is the vector of conserved quantities, $\mathbf{F}^{10}, \mathbf{G}^{10}$, and $\mathbf{H}^{10}$ are the flux vectors, and $\mathbf{S}^{10}$ is the source vector for the conservation form of the transport equations. These vectors are given by

$$
\begin{aligned}
& \mathbf{U}^{10}=\left(\begin{array}{c}
\rho \\
\rho u_{x} \\
\rho u_{y} \\
\rho u_{z} \\
\rho u_{x}^{2}+P_{x x} \\
\rho u_{x} u_{y}+P_{x y} \\
\rho u_{x} u_{z}+P_{x z} \\
\rho u_{y}^{2}+P_{y y} \\
\rho u_{y} u_{z}+P_{y z} \\
\rho u_{z}^{2}+P_{z z}
\end{array}\right), \quad \mathbf{F}^{10}=\left(\begin{array}{c}
\rho u_{x} \\
\rho u_{x}^{2}+P_{x x} \\
\rho u_{x} u_{y}+P_{x y} \\
\rho u_{x} u_{z}+P_{x z} \\
\rho u_{x}^{3}+3 u_{x} P_{x x} \\
\rho u_{x}^{2} u_{y}+2 u_{x} P_{x y}+u_{y} P_{x x} \\
\rho u_{x}^{2} u_{z}+2 u_{x} P_{x z}+u_{z} P_{x x} \\
\rho u_{x} u_{y}^{2}+u_{x} P_{y y}+2 u_{y} P_{x y} \\
\rho u_{x} u_{y} u_{z}+u_{x} P_{y z}+u_{y} P_{x z}+u_{z} P_{x y} \\
\rho u_{x} u_{z}^{2}+u_{x} P_{z z}+2 u_{z} P_{x z}
\end{array}\right) \\
& \mathbf{G}^{10}=\left(\begin{array}{c}
\rho u_{y} \\
\rho u_{x} u_{y}+P_{x y} \\
\rho u_{y}^{2}+P_{y y} \\
\rho u_{y} u_{z}+P_{y z} \\
\rho u_{x}^{2} u_{y}+2 u_{x} P_{x y}+u_{y} P_{x x} \\
\rho u_{x} u_{y}^{2}+u_{x} P_{y y}+2 u_{y} P_{x y} \\
\rho u_{x} u_{y} u_{z}+u_{x} P_{y z}+u_{y} P_{x z}+u_{z} P_{x y} \\
\rho u_{y}^{3}+3 u_{y} P_{y y} \\
\rho u_{y}^{2} u_{z}+2 u_{y} P_{y z}+u_{z} P_{y y} \\
\rho u_{y} u_{z}^{2}+u_{y} P_{z z}+2 u_{z} P_{y z}
\end{array}\right), \quad \mathbf{H}^{10}=\left(\begin{array}{c}
\rho u_{z} \\
\rho u_{x} u_{z}+P_{x z} \\
\rho u_{y} u_{z}+P_{y z} \\
\rho u_{z}^{2}+P_{z z} \\
\rho u_{x}^{2} u_{z}+2 u_{x} P_{x z}+u_{z} P_{x x} \\
\rho u_{x} u_{y} u_{z}+u_{x} P_{y z}+u_{y} P_{x z}+u_{z} P_{x y} \\
\rho u_{x} u_{z}^{2}+u_{x} P_{z z}+2 u_{z} P_{x z} \\
\rho u_{y}^{2} u_{z}+2 u_{y} P_{y z}+u_{z} P_{y y} \\
\rho u_{y} u_{z}^{2}+u_{y} P_{z z}+2 u_{z} P_{y z} \\
\rho u_{z}^{3}+3 u_{z} P_{z z}
\end{array}\right),
\end{aligned}
$$




$$
\mathbf{S}^{10}=-\frac{1}{\tau}\left(\begin{array}{c}
0 \\
0 \\
0 \\
0 \\
\left(2 P_{x x}-P_{y y}-P_{z z}\right) / 3 \\
P_{x y} \\
P_{x z} \\
\left(2 P_{y y}-P_{x x}-P_{z z}\right) / 3 \\
P_{y z} \\
\left(2 P_{z z}-P_{x x}-P_{y y}\right) / 3
\end{array}\right),
$$

where $\tau$ in the source term is a characteristic relaxation time related to viscosity and hydrostatic pressure:

$$
\tau=\frac{\mu}{p}
$$

with

$$
p=\frac{P_{i i}}{3}
$$

\section{Numerical scheme for PDE in conservation form}

Among numerical methods for hyperbolic system, those of the Godunov-type have been most successful; these require an algorithm for solving the Riemann problem arising at each cell interface, either exactly or approximately. For large system of equations it is practical to use an approximate Riemann solver that does not attempt to account for all separate waves through which the cells interact, but lumps the information. Harten, Lax, and Van Leer ${ }^{5}$ described two families of such methods; the latest member is due to Linde. ${ }^{6}$ The HLLL Riemann solver uses three waves to cover the domain of influence of the cell interface; it requires only the following knowledge:

- The PDE system is hyperbolic and possesses a convex entropy function;

- maximum and minimum wave speeds are known.

The 10-moment transport equations in compact form can be written as

$$
\frac{\partial \mathbf{U}}{\partial t}+\nabla \cdot \mathbf{F}(\mathbf{U})=\mathbf{S}(\mathbf{U})
$$

where $\mathbf{U}$ is the vector of conserved quantities, $\mathbf{F}$ is the 3 -D flux tensor and $\mathbf{S}$ is the source vector for the conservation form. This equation can be integrated over a fixed control volume $\mathcal{V}$; applying the divergence theorem to the flux integration yields

$$
\frac{\partial \overline{\mathbf{U}}}{\partial t}=-\frac{1}{\mathcal{V}} \oint_{\partial \mathcal{V}} \mathbf{F}_{\mathrm{n}}(\mathbf{U}) d s+\overline{\mathbf{S}}(\mathbf{U})
$$

where the overbar denotes a volume average and $\mathbf{F}_{\mathrm{n}}$ denotes the vector of outward fluxes normal to the surface element $d s$. Let $\mathbf{n}$ be the outward unit vector normal to $d s$, then $\mathbf{F}_{\mathbf{n}}=\mathbf{F} \cdot \mathbf{n}$. In general, evaluation of the volume-averaged source term $\overline{\mathbf{S}}(\mathbf{U})$ requires numerical quadrature since it is not equal to the source term evaluated at the averaged conserved variables. For example, in the 1-D case,

$$
\overline{\mathbf{S}}(\mathbf{U})_{i}=\mathbf{S}\left(\overline{\mathbf{U}}_{i}\right)+\mathcal{O}\left(\Delta x^{2}\right) .
$$

In a second-order accurate method such as described below, though, the average source term $\overline{\mathbf{S}}(\mathbf{U})_{i}$ can be replaced by $\mathbf{S}\left(\overline{\mathbf{U}}_{i}\right) \cdot{ }^{7}$ Finite-volume schemes of second-order accuracy in space can be written in semi-discrete form as

$$
\left(\frac{\partial \mathbf{U}_{i}}{\partial t}\right)^{k}=-\frac{1}{\mathcal{V}_{i}} \sum_{j \in \omega_{i}} \mathbf{F}_{\mathrm{n}}\left(\mathbf{U}_{i}^{k}, \mathbf{U}_{j}^{k}\right) s_{i j}+\mathbf{S}\left(\mathbf{U}_{i}^{k}\right),
$$


where $\omega_{i}$ is the set of indices of cells that share a face with cell $i, s_{i j}$ is the area of the face shared by cells $i$ and $j$, and $\mathcal{V}_{i}$ denotes the cell volume. Hancock's predictor-corrector version of the MUSCL scheme ${ }^{8,9}$ can be employed to solve system of partial differential equations in conservation form. Second-order accuracy in space and time is achieved by introducing linear subcell distributions and evaluating fluxes and source terms halfway during the time step. The half-time (predictor) step, which includes gradient-limiting, is done

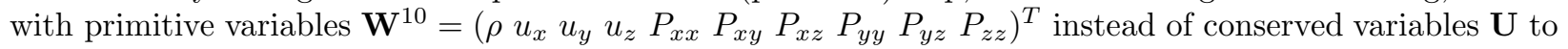
prevent non-physical values such as negative pressures. The half-time step can be written as

$$
\mathbf{W}_{i}^{k+\frac{1}{2}}=\mathbf{W}_{i}^{k}-\frac{\Delta t}{2 \mathcal{V}_{i}} \sum_{j \in \omega_{i}} \mathbf{A}_{\mathrm{n}} \tilde{\mathbf{W}}_{i}^{k} s_{i j}+\frac{\Delta t}{2} \mathbf{S}\left(\mathbf{W}_{i}^{k}\right),
$$

where $\mathbf{A}_{\mathrm{n}}$ denotes the coefficient matrix of the primitive equations, obtained by a similarity transformation of the flux Jacobian,

$$
\mathbf{A}_{\mathrm{n}}=\mathbf{M}^{-1}\left(\frac{\partial \mathbf{F}_{\mathrm{n}}}{\partial \mathbf{U}}\right) \mathbf{M}
$$

where

$$
\mathbf{M}=\frac{\partial \mathbf{U}}{\partial \mathbf{W}},
$$

and the tilde denotes the piecewise-linearly reconstructed value at the cell face,

$$
\tilde{\mathbf{W}}_{i}^{k}(\mathbf{r})=\mathbf{W}_{i}^{k}+\phi_{i}\left(\nabla \mathbf{W}^{k}\right)_{i} \cdot\left(\mathbf{r}-\mathbf{r}_{i}\right) .
$$

Here $\phi_{i}$ is a gradient limiter such as the double-minmod limiter ${ }^{10}$ and $\mathbf{r}_{i}$ is the centroid of cell $i$. The gradients of the primitive variables, $\nabla \mathbf{W}$, are obtained by solving least-square problems involving data from all adjacent cells. Once primitive variables at half-time are obtained, interface fluxes are computed by solving Riemann problems; the full-time (corrector) step to update conservative variables can be written as

$$
\mathbf{U}_{i}^{k+1}=\mathbf{U}_{i}^{k}-\frac{\Delta t}{\mathcal{V}_{i}} \sum_{j \in \omega_{i}} \mathbf{F}_{\mathrm{n}}\left(\tilde{\mathbf{W}}_{i}^{k+\frac{1}{2}}, \tilde{\mathbf{W}}_{j}^{k+\frac{1}{2}}\right) s_{i j}+\Delta t \mathbf{S}\left(\mathbf{W}_{i}^{k+\frac{1}{2}}\right),
$$

where

$$
\tilde{\mathbf{W}}_{i}^{k+\frac{1}{2}}(\mathbf{r})=\mathbf{W}_{i}^{k+\frac{1}{2}}+\phi_{i}\left(\nabla \mathbf{W}^{k}\right)_{i} \cdot\left(\mathbf{r}-\mathbf{r}_{i}\right) .
$$

\section{Time step for hyperbolic system with stiff source term}

Finding the allowable time step for a highly nonlinear system of equations on general computational meshes is not straightforward. In the case of the moment equations, the presence of distinct characteristic time scales, the advection time scale and the relaxation time scale due to the source term, makes the analysis even more difficult. In practice, an analogy to the result from a simple 1-D problem may be applied to the multidimensional problem ${ }^{11}$ the stability limit for explicit time integration in cell $i$ is approximately given by

$$
\Delta t_{i} \leq \frac{\mathcal{V}_{i}}{\sum_{j \in \omega_{i}}\left|\lambda_{i j}\right|^{\max } s_{i j}+\mathcal{V}_{i} / \tau_{i}},
$$

where $\left|\lambda_{i j}\right|^{\text {max }}$ is the largest wave speed on either side of the cell face $(i, j)$ and $\tau_{i}$ is the relaxation time in cell $i$. It shows that the local time step is determined by the combination of two characteristic times, $\Delta n / \lambda$ and $\tau$, where $\Delta n=\mathcal{V}_{i} / s_{i j}$ is the width of cell $i$ normal to $s_{i j}$. This criterion is restrictive, especially when the flow field is near the equilibrium where the relaxation time is much smaller than the advection time. Our main interest is in wave propagation; however, the time step has to be of the same order of the relaxation time to resolve the correct physics. Currently, part of our research activity is in developing numerical methods that capture the relaxation physics correctly, even though a large (advection-based) time step is used. This will be discussed further in Section VII. 


\section{HLLL Riemann solver for the 10-moment model}

This solver is designed to capture an isolated discontinuity exactly, and do a reasonable job if more waves are present. This simple design criterion allows us to approximate the solution of a Riemann problem by only three waves bracketing two intermediate states. For the 1-D Euler equations, all approximate Riemann solvers based on characteristic decomposition use three waves anyway, but more complicated physical systems such as magnetohydrodynamics, radiation hydrodynamics, and extended-hydrodynamics posses more than three waves, and characteristic-based solvers need to distinguish all waves in order to provide a detailed approximation. In the three-wave HLL Riemann solver the middle wave speed, representing an isolated discontinuity, is obtained by solving generalized Rankine-Hugoniot conditions instead of using known analytical formula for the wave speeds. Thus, the algorithm does not require a full analysis of the characteristic wave decomposition for the system of PDE's. As Linde mentions, the family of HLL Riemann solvers can be applied to complex physical systems for which the characteristic wave-decomposition analysis is extremely difficult. ${ }^{6}$ In this respect, system of extended-hydrodynamics equations are excellent candidates. In fact, the eigenstructure of the 10-moment model was already analyzed by Brown et al., ${ }^{12,13}$ and analytical results are known. However, its simplicity and the planned application of the algorithm to even higher-order moment models such as the 35 -moment model equations ${ }^{12,14}$ made us select the HLLL Riemann solver to compute the cell-interface fluxes.

The middle wave speed $V$ is obtained in the least-square sense,

$$
V=\frac{(\Delta \mathbf{U}, \Delta \mathbf{F})_{\mathrm{P}}}{\|\Delta \mathbf{U}\|_{\mathrm{P}}^{2}}=\frac{\Delta \mathbf{U}^{T} \mathbf{P} \Delta \mathbf{F}}{\Delta \mathbf{U}^{T} \mathbf{P} \Delta \mathbf{U}}=\frac{\Delta \mathbf{W}^{T} \Delta \mathbf{F}}{\Delta \mathbf{W}^{T} \Delta \mathbf{U}},
$$

where

$$
\mathbf{W}(\mathbf{U})=\frac{\partial S(\mathbf{U})}{\partial \mathbf{U}}
$$

is a vector of symmetrizing variables (not primitive variables here), formed by taking derivatives of the entropy function $S(\mathbf{U})$. The symmetric positive-definite matrix $\mathbf{P}$ is the Hessian of $S(\mathbf{U})$,

$$
\mathbf{P}(\mathbf{U})=\frac{\partial^{2} S(\mathbf{U})}{\partial \mathbf{U}^{2}}=\frac{\partial \mathbf{W}(\mathbf{U})}{\partial \mathbf{U}}
$$

The entropy function of the 10-moment model is given as

$$
S(\mathbf{U})=-\rho\left(\frac{1}{3} \ln \frac{\operatorname{det} \boldsymbol{\Theta}}{\rho^{2}}\right) .
$$

Straightforward differentiation of the entropy function produces the symmetrizing variables $\mathbf{W}$ and (for later use) the diagonal entries of the matrix $\mathbf{P}$,

$$
\mathbf{W}(\mathbf{U})=\frac{2}{3}\left(\begin{array}{c}
\frac{1}{2}\left(5-\ln \frac{\operatorname{det} \boldsymbol{\Theta}}{\rho^{2}}-\boldsymbol{u}^{T} \boldsymbol{\Phi} \boldsymbol{u}\right) \\
\boldsymbol{\phi}_{1}^{T} \boldsymbol{u} \\
\boldsymbol{\phi}_{2}^{T} \boldsymbol{u} \\
\boldsymbol{\phi}_{3}^{T} \boldsymbol{u} \\
-\frac{1}{2} \Phi_{11} \\
-\Phi_{12} \\
-\Phi_{13} \\
-\frac{1}{2} \Phi_{22} \\
-\Phi_{23} \\
-\frac{1}{2} \Phi_{33}
\end{array}\right), \quad \operatorname{diag}[\mathbf{P}(\mathbf{U})]=\frac{2}{3 \rho}\left(\begin{array}{c}
\frac{1}{2}\left(\left(\boldsymbol{u}^{T} \boldsymbol{\Phi} \boldsymbol{u}\right)^{2}+5\right) \\
\left(\phi_{1}^{T} \boldsymbol{u}\right)^{2}+\left(1+\boldsymbol{u}^{T} \boldsymbol{\Phi} \boldsymbol{u}\right) \Phi_{11} \\
\left(\phi_{2}^{T} \boldsymbol{u}\right)^{2}+\left(1+\boldsymbol{u}^{T} \boldsymbol{\Phi} \boldsymbol{u}\right) \Phi_{22} \\
\left(\phi_{3}^{T} \boldsymbol{u}\right)^{2}+\left(1+\boldsymbol{u}^{T} \boldsymbol{\Phi} \boldsymbol{u}\right) \Phi_{33} \\
\frac{1}{2} \Phi_{11}^{2} \\
\Phi_{12}^{2}+\Phi_{11} \Phi_{22} \\
\Phi_{13}^{2}+\Phi_{11} \Phi_{33} \\
\frac{1}{2} \Phi_{22}^{2} \\
\Phi_{23}^{2}+\Phi_{22} \Phi_{33} \\
\frac{1}{2} \Phi_{33}^{2}
\end{array}\right)
$$

where

$$
\mathbf{\Phi} \equiv \boldsymbol{\Theta}^{-1}=\left[\begin{array}{lll}
\phi_{1} & \phi_{2} & \phi_{3}
\end{array}\right], \quad \Phi_{i j} \equiv \Theta_{i j}^{-1}, \quad i, j \in\{1,2,3\},
$$

and $\boldsymbol{u}$ is the velocity vector. Once the symmetrizing variables are obtained, the middle wave speed can be computed by Eq.(20); note that the matrix $\mathbf{P}$ is not explicitly needed here. Then cell-interface fluxes are 
obtained by

$$
\mathbf{F}_{\mathrm{n}}\left(\mathbf{U}_{i}, \mathbf{U}_{j}\right)=\frac{\lambda_{+} \mathbf{F}_{\mathrm{n}}\left(\mathbf{U}_{i}\right)-\lambda_{-} \mathbf{F}_{\mathrm{n}}\left(\mathbf{U}_{j}\right)}{\lambda_{+}-\lambda_{-}}+\frac{(1-\alpha) \lambda_{-} \lambda_{+}+\alpha\left(\lambda_{-} V_{+}+\lambda_{+} V_{-}\right)}{\lambda_{+}-\lambda_{-}} \Delta \mathbf{U},
$$

where

$$
\begin{aligned}
\lambda_{\max , \min } & =\mathbf{n}^{T} \boldsymbol{u} \pm \sqrt{3 \mathbf{n}^{T} \boldsymbol{\Theta} \mathbf{n}}, \\
\lambda_{+} & =\max \left(0, V, \lambda_{\max }\left(\mathbf{U}_{i}\right), \lambda_{\max }\left(\mathbf{U}_{j}\right)\right), \quad \lambda_{-}=\min \left(0, V, \lambda_{\min }\left(\mathbf{U}_{i}\right), \lambda_{\min }\left(\mathbf{U}_{j}\right)\right), \\
V_{+} & =\max (0, V), \quad V_{-}=\min (0, V) .
\end{aligned}
$$

Recall $\mathbf{n}$ is an outward unit vector normal to the cell face. The parameter $\alpha \in[0,1]$ is an estimation of the relative strength of the middle wave. The computation of $\alpha$ requires knowledge of the matrix $\mathbf{P}$,

$$
\alpha=H\left(V \Delta S-\Delta\left(\mathbf{n}^{T} \boldsymbol{u} S\right)\right) \frac{(\Delta \mathbf{U}, \Delta \mathbf{F})_{\mathrm{P}}^{2}}{\|\Delta \mathbf{U}\|_{\mathrm{P}}^{2}\|\Delta \mathbf{F}\|_{\mathrm{P}}^{2}},
$$

where $H(x)$ is the Heaviside step function preventing violation of the entropy condition,

$$
H(x)=\left\{\begin{array}{ll}
0 & x<0 \text { (discontinuity violates the entropy condition) } \\
1 & x \geq 0 \text { (entropy inequality satisfied) }
\end{array} .\right.
$$

In practice we may reduce $\mathbf{P}$ to its main diagonal, hence the need for Eq.(24).

\section{Numerical results}

\section{A. Resolving 1-D shock structure}

We present some 1-D results from validation studies in which we tried to produce steady shock profiles for various inflow Mach numbers. Assuming a steady state leads to a system of ordinary differential equations (ODE), which can be solved by a standard fourth-order Runge-Kutta method. ${ }^{12}$ The resulting ODE solutions are compared with the solution of the PDE's obtained by the finite-volume method described in the previous section. Upstream and downstream boundary conditions are assumed to be in equilibrium; given the upstream Mach number, density, and velocity, downstream conditions are determined from the jump-equations. Two different upstream Mach numbers $\left(\mathrm{M}_{U}=1.1,5.0\right)$ representing weak-and strongshock cases, are examined. To avoid constraints by upstream and downstream values, a sufficiently wide computational domain is taken. The computational domain based on the upstream mean free path is shown in Table 2. We assume the monatomic gas is Argon $\left(\mathrm{MW}_{\mathrm{Ar}}=39.948 \mathrm{~kg} / \mathrm{kmol}\right)$ and the power law is used for the viscosity,

$$
\frac{\mu}{\mu_{\text {ref }}}=\left(\frac{T}{T_{\text {ref }}}\right)^{n}
$$

where $\mu_{\text {ref }}=2.125 \times 10^{-5} \mathrm{Ns} / \mathrm{m}^{2}, T_{\text {ref }}=273 \mathrm{~K}$, and $n=13 / 18$ for Argon. ${ }^{12,15}$ Density distributions are normalized by the upstream and downstream density using following formula,

$$
\hat{\rho}=\frac{\rho-\rho_{U}}{\rho_{D}-\rho_{U}},
$$

and shown in Figures 1 and 2 superimposed on ODE results. The spatial dimension is normalized by the upstream mean free path derived in gas-kinetic theory using an elastic, hard-sphere model, ${ }^{16}$

$$
l_{U}=\frac{16 \mu}{5(2 \pi \rho p)^{1 / 2}} .
$$

The PDE-based solutions (symbols) agree well with the solutions obtained by integrating the ODE's describing the steady structures (solid line). Using the ODE-based solutions as a benchmark $\left(\mathrm{M}_{U}=1.1\right)$, density errors were computed for a sequence of grids; its convergence rate demonstrates the second-order spatial accuracy of the method used (Table 3). 
Table 2. Initial condition and problem setup in each test problems.

\begin{tabular}{ccccccc}
\hline & $\mathrm{M}_{U}$ & $l_{U}[\mathrm{~m}]$ & $\rho_{U}$ & $\rho_{D}$ & number of cells & computational domain \\
\hline test1 & 1.1 & $8.549 \times 10^{-9}$ & 1.0 & 1.150 & 200 & $x / l_{U} \in[-100,100]$ \\
test2 & 5.0 & $4.362 \times 10^{-9}$ & 1.0 & 3.571 & 200 & $x / l_{U} \in[-20,20]$ \\
\hline
\end{tabular}

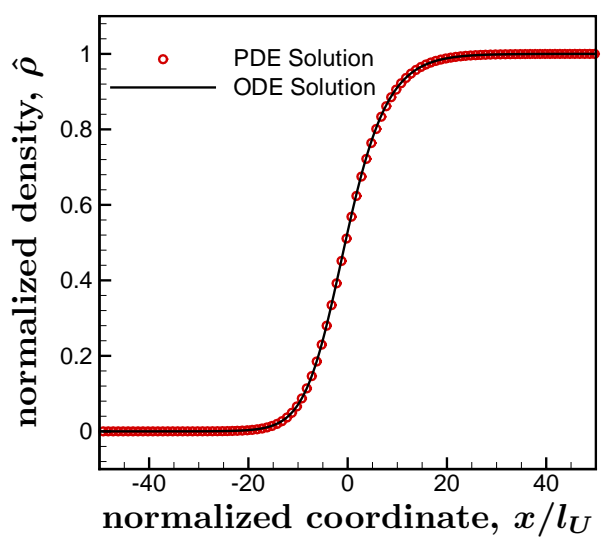

Figure 1. Density distribution in steady shock structure for $\mathrm{M}_{U}=1.1$. The space coordinate is normalized by the upstream mean free path $l_{U}$.

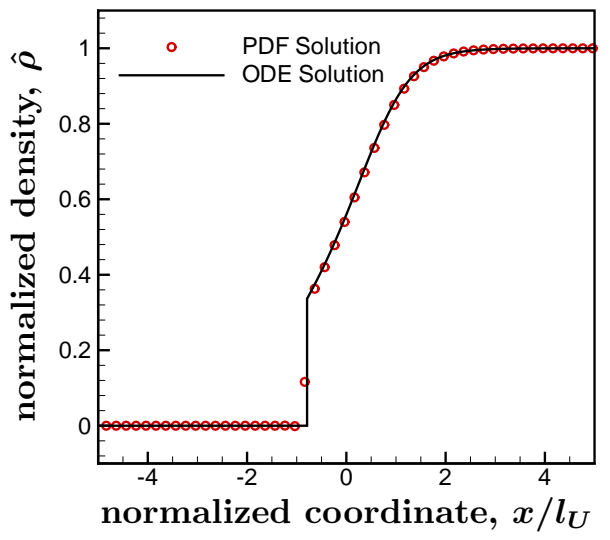

Figure 2. Density distribution in steady shock structure for $M_{U}=5.0$. A "frozen" shock is followed by a relaxation zone.

Table 3. Grid convergence study of error norms shows that steady shock solutions $\left(\mathrm{M}_{U}=1.1\right)$ are second-order accurate.

\begin{tabular}{ccccccc}
\hline number of cells & $L_{1}(\rho)$ & order & $L_{2}(\rho)$ & order & $L_{\infty}(\rho)$ & order \\
\hline 50 & $5.168 \mathrm{e}-03$ & - & $1.352 \mathrm{e}-02$ & - & $6.174 \mathrm{e}-02$ & - \\
100 & $1.277 \mathrm{e}-03$ & 2.02 & $3.730 \mathrm{e}-03$ & 1.86 & $1.753 \mathrm{e}-02$ & 1.82 \\
200 & $3.084 \mathrm{e}-04$ & 2.05 & $9.141 \mathrm{e}-04$ & 2.03 & $4.281 \mathrm{e}-03$ & 2.03 \\
400 & $7.525 \mathrm{e}-05$ & 2.04 & $2.220 \mathrm{e}-04$ & 2.04 & $1.019 \mathrm{e}-03$ & 2.07 \\
\hline
\end{tabular}

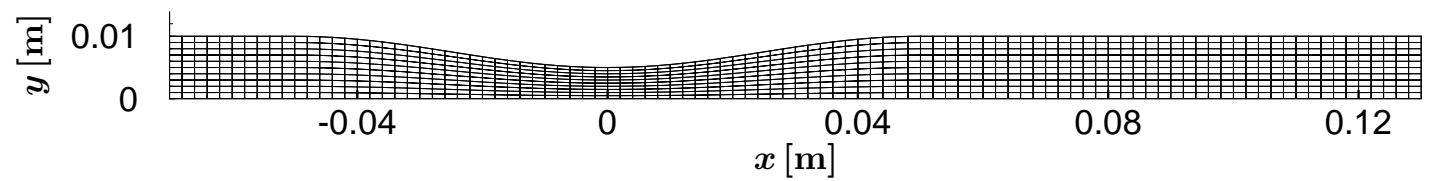

Figure 3. Computational grid of cosine curve nozzle. Number of cells are $100 \times 10$. 


\section{B. Cosine-nozzle flow}

Internal nozzle flow is examined as the first 2-D test case. Since there is no stagnation point inside the nozzle, this flow problem is easier than an airfoil problem and serves as a precursor test case. A symmetric cosine-shaped nozzle (Figure 3) is used as the computational domain. The throat is located at the origin of the $x$-axis and the total length over which area variation occurs is $0.1 \mathrm{~m}$. There are $0.02 \mathrm{~m}$ and $0.08 \mathrm{~m}$ long constant-area regions at inlet and outlet. Table 4 shows the reservoir conditions. Stagnation temperature

Table 4. Reservoir condition for the nozzle flow

\begin{tabular}{ccccccc}
\hline $\mathrm{Re}$ & $\mathrm{Kn}$ & $\rho_{0}\left[\mathrm{~kg} / \mathrm{m}^{3}\right]$ & $p_{0}[\mathrm{~Pa}]$ & $T_{0}[\mathrm{~K}]$ & $h_{0}[\mathrm{~J} / \mathrm{kg}]$ & $r_{t}[\mathrm{~m}]$ \\
\hline 100 & 0.014 & $8.229 \times 10^{-4}$ & 51.38 & 300 & $1.561 \times 10^{5}$ & $5.0 \times 10^{-3}$ \\
\hline
\end{tabular}

$T_{0}$ and Reynolds number are specified in the reservoir. The Knudsen number is based on the throat width and the reservoir condition. The Reynolds number is defined as ${ }^{17}$

$$
\operatorname{Re}=\frac{\rho_{0} \sqrt{2 h_{0}} r_{t}}{\mu_{0}}
$$

where $\sqrt{2 h_{0}}$ is an ideal maximum escape speed from the reservoir and $r_{t}$ is the throat half-width. Equation (33) leads to a direct relation between Reynolds number and reservoir pressure,

$$
p_{0}=\left(\sqrt{\frac{\gamma-1}{2 \gamma} R T_{0}} \frac{\mu_{0}}{r_{t}}\right) \text { Re. }
$$

In this test case, Argon $\left(\mu_{0}=2.299 \times 10^{-5} \mathrm{Ns} / \mathrm{m}^{2}, R_{\mathrm{Ar}}=208.13 \mathrm{~J} / \mathrm{kg} \mathrm{K}\right)$ is used and the reservoir pressure satisfies the relation

$$
p_{0} \simeq 0.5138 \text { Re. }
$$

Viscosity is computed by Sutherland's law,

$$
\frac{\mu}{\mu_{\text {ref }}}=\left(\frac{T}{T_{\text {ref }}}\right)^{3 / 2} \frac{T_{\text {ref }}+S}{T+S}
$$

where $\mu_{\text {ref }}=2.125 \times 10^{-5} \mathrm{Ns} / \mathrm{m}^{2}, T_{\text {ref }}=273 \mathrm{~K}$, and $S=144 \mathrm{~K}$ for Argon. ${ }^{15}$ Figure 4 shows the normalized

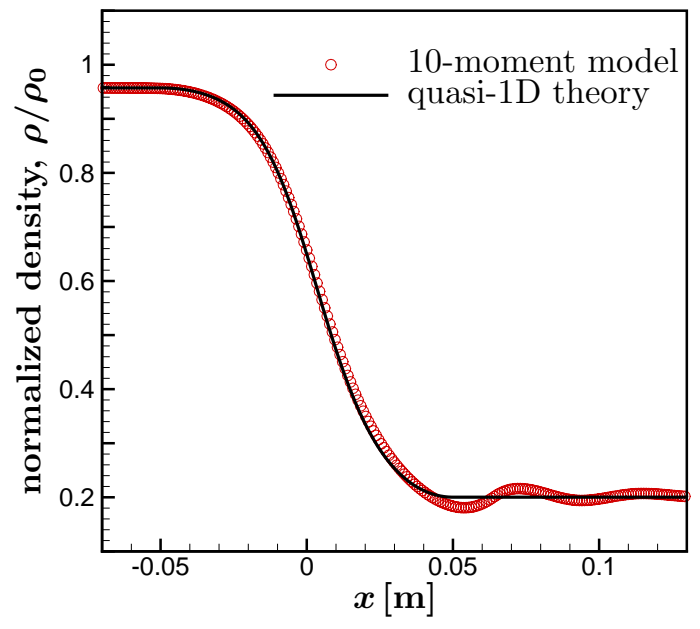

Figure 4. Density profile along axis of nozzle.

density profile obtained by the 10-moment model (circles) on the axis direction, compared with quasi-1D theory (solid line). There is a good agreement between the PDE solution and the theoretical density profile. The decay of the (diamond-pattern) waves in the supersonic section indicate that the equation system is dissipative (unlike the Euler equations). 


\section{NACA0012 Micro-Airfoil flow}

Next, the external flow around a NACA0012 micro-airfoil is computed using the 10-moment model. The free-stream initial conditions are show in Table 5. The Knudsen number is based on the chord length of the airfoil and the free-stream condition. The chord length of the airfoil is $0.04 \mathrm{~m}$ and a $C$-type grid is used. The grid geometry is shown in Figure 5. Since various results are available using air as the gas, we also assume the gas is air $\left(\mathrm{MW}_{\text {Air }}=28.966 \mathrm{~kg} / \mathrm{kmol}\right)$, even though the 10 -moment model assumes a monatomic gas. Viscosity is computed by Sutherland's law (Eq.(36)) where $\mu_{\text {ref }}=1.716 \times 10^{-5} \mathrm{Ns} / \mathrm{m}^{2}, T_{\text {ref }}=273 \mathrm{~K}$, and $S=111 \mathrm{~K}$ for Air. Hittinger has added rotational degrees of freedom to the system, leading to an 11-moment model for a diatomic gas; ${ }^{18}$ we have not used this model. The 10 -moment equation implicitly assume $\gamma=5 / 3$, rather than $\gamma=1.4$; for the density results presented below this hardly makes a difference (see below).

The 10-moment result is shown in Figure 6 with the corresponding Sun-Boyd result ${ }^{2}$ reproduced in Figure 8. In this continuum-transition regime, the flow on the wall has a finite velocity. This slip velocity is given by Maxwell's first-order slip boundary condition

$$
u_{\text {gas }}-u_{\text {wall }}=\left.\frac{2-\sigma}{\sigma} l \frac{\partial u_{t}}{\partial n}\right|_{w},
$$

where $\sigma$ is an accommodation coefficient and $u_{t}$ is the tangential component of velocity at the wall. At the airfoil, completely diffuse molecular reflection is assumed in formulating the boundary condition for both methods (achieved by setting $\sigma=1$ ). There are clear differences between the solutions, especially upstream of the airfoil. Near the stagnation point the 10-moment approach gives significantly lower density values than the DSMC/NS approach, with the former values expected to be the more accurate ones. This is borne out by the experimental results ${ }^{19}$ reproduced in Figure 9. Based on the isentropic relation, the stagnation density for the given upstream Mach number, compared for a monatomic gas and for a diatomic gas, differ only by $1.1 \%\left(\left(\rho_{0} / \rho_{\infty}\right)_{\gamma=5 / 3}=1.337,\left(\rho_{0} / \rho_{\infty}\right)_{\gamma=1.4}=1.351\right)$; thus we conclude that the monatomic gas assumption in the 10-moment model does not affect the density distribution much in the case considered.

Despite the good agreement with the experiment near the leading edge, the normalized density near the trailing edge is slightly higher than the experimental value. This might be improved upon by developing a new set of boundary conditions for the 10-moment model, more accurate than Maxwell's first-order slip model. Navier-Stokes results using the first-order slip boundary condition are shown in Figure 7; they are closer to the DSMC/NS results than to the 10-moment or experimental results. The 10-moment results are obtained in about one third the time it takes the DSMC/NS result to converge; a much greater efficiency gain is still in store.

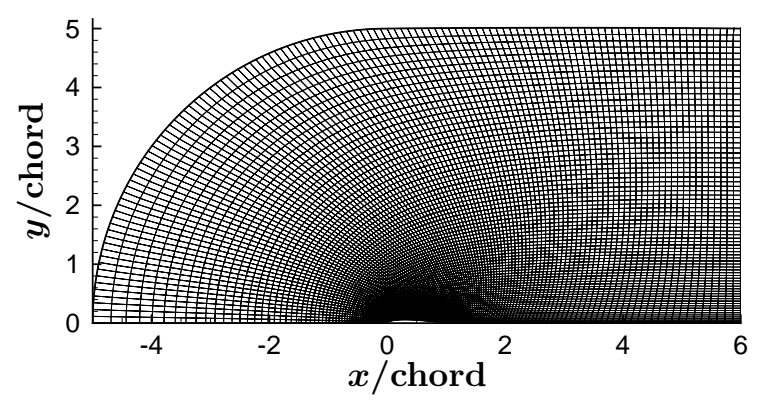

Figure 5. Computational grid around NACA0012 micro-airfoil. The coordinate is normalized by the chord length. The number of cells is $120 \times 76$.

Table 5. Free stream condition for micro-airfoil flow

\begin{tabular}{ccccccc}
\hline $\mathrm{M}_{\infty}$ & $\operatorname{Re}_{\infty}$ & $\mathrm{Kn}_{\infty}$ & $\rho_{\infty}\left[\mathrm{kg} / \mathrm{m}^{3}\right]$ & $U_{\infty}[\mathrm{m} / \mathrm{s}]$ & $T_{\infty}[\mathrm{K}]$ & $L_{\text {chord }}[\mathrm{m}]$ \\
\hline 0.8 & 73 & 0.017 & $1.161 \times 10^{-4}$ & 257 & 257 & 0.04 \\
\hline
\end{tabular}




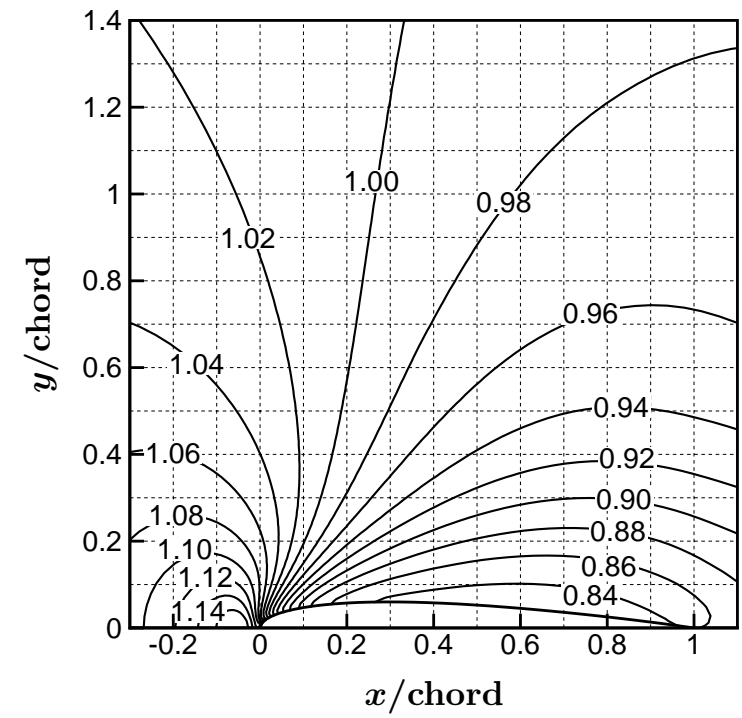

Figure 6. Density distribution $\left(\rho / \rho_{0}\right)$ around NACA 0012 airfoil by the 10-moment model

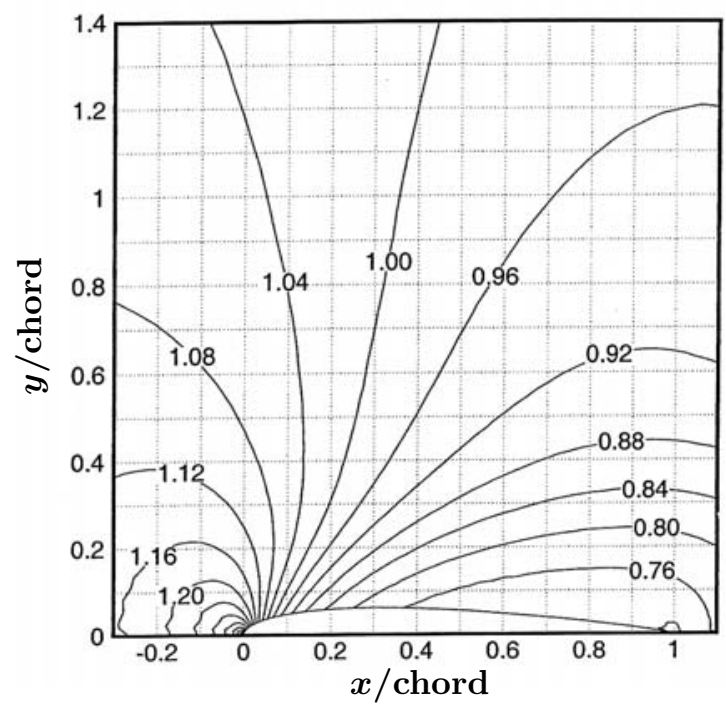

Figure 8. Density distribution $\left(\rho / \rho_{0}\right)$ around NACA 0012 airfoil by the IP method

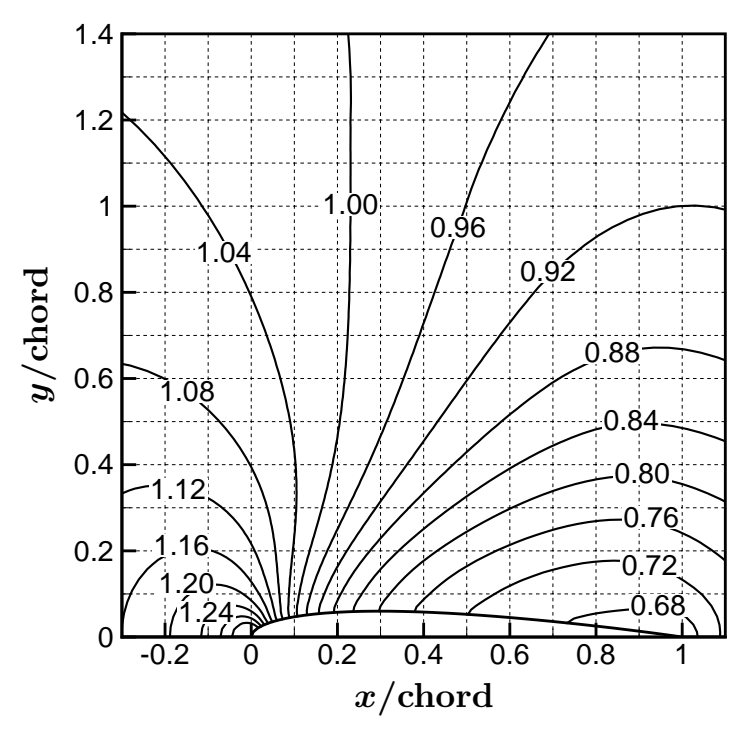

Figure 7. Density distribution $\left(\rho / \rho_{0}\right)$ around NACA 0012 airfoil by the Navier-Stokes equations

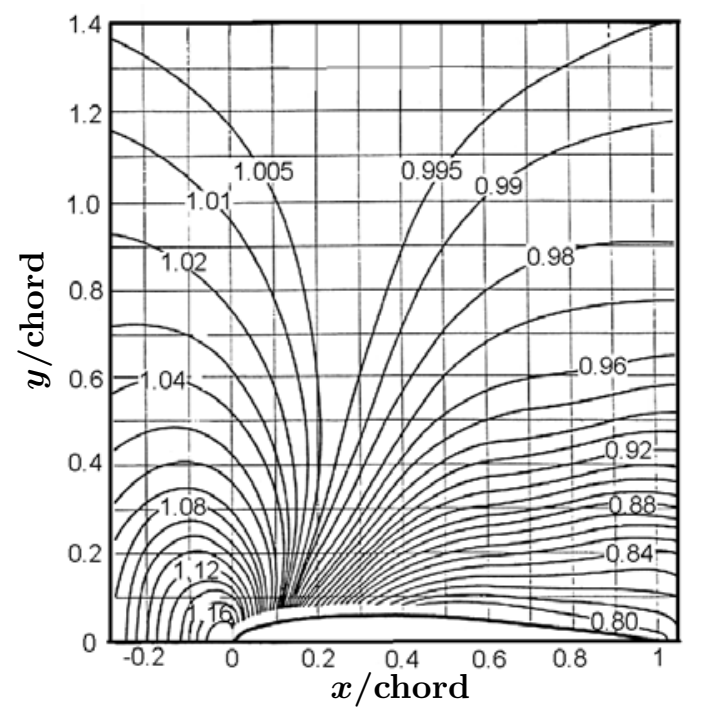

Figure 9. Density distribution $\left(\rho / \rho_{0}\right)$ around NACA 0012 airfoil by the experiment

\section{Asymptotic preserving methods}

So far we have applied a conventional Godunov-type finite volume method (FVM) to the 10-moment model. Numerical results show for steady shock of Figure 1 that the PDE is solved successfully, with second-order accuracy in space. However, the presence of the source term, which contains the relaxation time $\tau$, raises the numerical challenge. As mentioned earlier, the time step for explicit time-marching is restricted by the smaller of the advection and relaxation time scales. When we are interested in resolving the actual relaxation process, for instance when resolving the shock structure $(\Delta x \simeq \mathcal{O}(l))$, this time step is not restrictive. In this case, advection time and relaxation time are same order. Numerical difficulty arises when the relaxation process is under-resolved $(\Delta x \gg \mathcal{O}(l))$. In this limit, we would prefer to take a large time step $(\Delta t \gg \mathcal{O}(\tau))$ which is of the order of the advection time scale or cell size. This will cause loss of numerical accuracy, since the flux function can not capture the contribution of the source term in a large 
time step. Basically, the flux function does not have any mechanism of relaxation. Thus, our current method has to take the time step of the same order as for the relaxation time (typically $\Delta t=10^{-7}-10^{-9}$ ) in order to couple the relaxation process to the flux function. It is obvious that for reaching a steady state, especially near equilibrium $(\tau \rightarrow 0)$, a large number of iterations is required.

A numerical method is called "asymptotic-preserving" (AP) if it can capture the relaxation process correctly while taking $\Delta t \gg \mathcal{O}(\tau)$ and $\Delta x \gg \mathcal{O}(l) .{ }^{20}$ Development of AP methods has become an active research area in recent years. ${ }^{18,21-23}$ One promising method is a particular semi-implicit Discontinuous Galerkin (DG) method that has been shown to have the AP property for the 1-D generalized hyperbolic heat equation (GHHE) ${ }^{24}$ The DG method is a hybrid of the finite-element discretization and the FVM. The methods are identical in the first-order case; in the second-order case, the main difference is that the DG method updates both cell-averaged values and slope values simultaneously, whereas FVM updates only cell averages and derives slopes afterwards by finite differencing. It seems that the accuracy is maintained in the under-resolved limit because the evolution of the solution slope is computed directly from an equation that accounts for the effect of the source term. Since these slope values are used to compute input values for the flux function, the flux function feels the influence of the relaxation mechanism. Recently a semi-implicit DG method has been shown to have the AP property only for a 1-D linear system of equations. Further analysis of multidimensional problems and nonlinear equations is under way. ${ }^{25}$

\section{Conclusion}

In this paper we use the 10-moment description of fluid flow to compute internal nozzle flow and external flow around a micro-airfoil in the continuum-transition regime. The numerical method used is Hancock's scheme, a second-order non-oscillatory Godunov-type scheme; the numerical flux function incorporated is of the Harten-Lax-van Leer (HLL) type and due to Linde (HLLL). Maxwell's first-order slip velocity model is employed to prescribe the slip velocity on the wall face. The numerical solutions are validated by a comparison to results obtained with a hybrid DSMC/Navier-Stokes method and an experiment. The results shows good agreement with the experiments near the leading edge (stagnation point) where the Gaussian distribution function recovers the equilibrium Maxwellian distribution function. However, the density profile on the airfoil has slightly higher downstream values than the experiment. To improve this result, a rigorous analysis and derivation of boundary conditions for the 10-moment model may be required; in addition, it may be necessary to step up to the 11-moment model, which includes a separate rotational temperature.

\section{Acknowledgement}

This work was supported by Grant Nr. F49620-03-1-0226 from the Air Force Office of Scientific Research.

\section{References}

\footnotetext{
${ }^{1}$ Agarwal, R. K., Yun, K.-Y., and Balakrishnan, R., "Beyond Navier-Stokes: Burnett Equations for Flows in the Continuum-Transition Regime," Physics of Fluids, Vol. 13, No. 10, October 2001, pp. 3061-3085.

${ }^{2}$ Sun, Q. and Boyd, I. D., "A Direct Simulation Method for Subsonic, Microscale Gas Flows," Journal of Computational Physics, Vol. 179, 2002, pp. 400-425.

${ }^{3}$ Sun, Q., Boyd, I. D., and Candler, G. V., "A Hybrid Continuum/Particle Approach for Modeling Subsonic, Rarefied Gas Flows," Journal of Computational Physics, Vol. 194, No. 1, 2004, pp. 256-277.

${ }^{4}$ Levermore, C. D., "Moment Closure Hierarchies for Kinetic Theories," Journal of Statistical Physics, Vol. 83, No. 5-6, 1996, pp. 1021-1065.

${ }^{5}$ Harten, A., Lax, P. D., and van Leer, B., "On Upstream Differencing and Godunov-Type Schemes for Hyperbolic Conservation Laws," SIAM Review, Vol. 25, No. 1, Jan. 1983, pp. 35-61.

${ }^{6}$ Linde, T., "A Practical, General-Purpose, Two-State HLL Riemann Solver for Hyperbolic Conservation Laws," International Journal for Numerical Methods in Fluids, Vol. 40, 2002, pp. 391-402.

${ }^{7}$ Pareschi, L. and Russo, G., "Implicit-Explicit Runge-Kutta Schemes and Applications to Hyperbolic Systems with Relaxation," University of Ferrara, Ferrara, Italy, 2004 (unpublished).

${ }^{8}$ van Albada, G. D., van Leer, B., and Roberts, Jr., W. W., "A Comparative Study of Computational Methods in Cosmic Gas Dynamics," Astronomy and Astrophysics, Vol. 108, No. 1, April 1982, pp. 76-84.

${ }^{9}$ van Leer, B., "Upwind and High-Resolution Methods for Compressible Flow: From Donor Cell to Residual-Distribution Schemes," AIAA-2003-3559, 16th AIAA Computational Fluid Dynamics Conference, Orlando, Florida, June 2003.

${ }^{10}$ Barth, T. J., "On Unstructured Grids and Solvers," Lecture Series 1990-03, Vol.2, von Karman Institute for Fluid Dynamics, March 1990.
} 
${ }^{11}$ Linde, T. J., A Three-Dimensional Adaptive Multifluid MHD Model of the Heliosphere, Ph.D. thesis, University of Michigan, Ann Arbor, Michigan, 1998.

${ }^{12}$ Brown, S. L., Approximate Riemann Solvers for Moment Models of Dilute Gases, Ph.D. thesis, University of Michigan, Ann Arbor, Michigan, 1996.

${ }^{13}$ Brown, S. L., Roe, P. L., and Groth, C. P. T., "Numerical Solution of a 10-Moment Model for Nonequilibrium Gasdynamics," AIAA-1995-1677, 12th AIAA Computational Fluid Dynamics Conference, San Diego, California, June 1995.

${ }^{14}$ Groth, C. P. T., Roe, P. L., Gombosi, T. I., and Brown, S. L., "On the Nonstationary Wave Structure of a 35-Moment Closure for Rarefied Gas Dynamics," AIAA-1995-2312, 12th AIAA Computational Fluid Dynamics Conference, San Diego, California, June 1995.

${ }^{15}$ White, F. M., Viscous Fluid Flow, McGraw-Hill, Inc., 1991.

${ }^{16}$ Bird, G. A., Molecular Gas Dynamics and the Direct Simulation of Gas Flows, Oxford University Press, 1994.

${ }^{17}$ Rothe, D. E., "Electron-Beam Studies of Viscous Flows in Supersonic Nozzles," AIAA Journal, Vol. 9, No. 5, 1971, pp. 804-811.

${ }^{18}$ Hittinger, J. A. F., Foundations for the Generalization of the Godunov Method to Hyperbolic Systems with Stiff Relaxation Source Terms, Ph.D. thesis, University of Michigan, Ann Arbor, Michigan, 2000.

${ }^{19}$ Allegre, J., Raffin, M., and Lengrand, J. C., "Experimental Flowfields Around NACA 0012 Airfoils Located in Subsonic and Supersonic Rarefied Air Streams," Numerical Simulation of Compressible Navier-Stokes Flows, edited by M. O. Bristeau, R. Glowinski, J. Periaux, and H. Viviand, Vol. 18 of Notes on Numerical Fluid Mechanics, Friedr. Vieweg and Sohn, Braunschweig, Germany, 1987, pp. 59-68.

${ }^{20}$ Jin, S., "Efficient Asymptotic-Preserving (AP) Schemes for Some Multiscale Kinetic Equations," SIAM Journal on Scientific Computing, Vol. 21, No. 2, September-October 1999, pp. 441-454.

${ }^{21}$ Arora, M., Explicit Characteristic-Based High-Resolution Algorithms for Hyperbolic Conservation Laws with Stiff Source Terms, Ph.D. thesis, University of Michigan, Ann Arbor, Michigan, 1996.

${ }^{22}$ Jin, S. and Levermore, C. D., "Numerical Schemes for Hyperbolic Conservation Laws with Stiff Relaxation Terms," Journal of Computational Physics, Vol. 126, No. 2, 1996, pp. 449-467.

${ }^{23}$ Liotta, S. F., Romano, V., and Russo, G., "Central Schemes for Balance Laws of Relaxation Type," SIAM Journal on Numerical Analysis, Vol. 38, No. 4, 2000, pp. 1337-1356.

${ }^{24}$ Lowrie, R. B. and Morel, J. E., "Methods for Hyperbolic Systems with Stiff Relaxation," International Journal for Numerical Methods in Fluids, Vol. 40, No. 3-4, September 2002, pp. 413-423.

${ }^{25}$ Hittinger, J. A. F., Suzuki, Y., and van Leer, B., "Investigation of the Discontinuous Galerkin Method for First-Order PDE Approaches to CFD," submitted to, 17th AIAA Computational Fluid Dynamics Conference, Toronto, Ontario, June 2005. 